\title{
Beam halo in high-intensity hadron accelerators caused by statistical gradient er rors
}

\author{
Frank Gerigk* \\ ASTeC, Intense Beams Group, Rutherford Appleton Laboratory, Chilton, Didcot, OXON, OX11 OQX, United Kingdom
}

(Received 3 February 2004; published 9 June 2004)

\begin{abstract}
The particle-core model for a continuous cylindrical beam is used to describe the motion of single particles oscillating in a uniform linear focusing channel. Using a random variation of the focusing forces, the model is deployed as proof of principle for the occurrence of large single particle radii without the presence of initial mismatch of the beam core. Multiparticle simulations of a periodic 3D transport channel are then used to qualify and quantify the effects in a realistic accelerator lattice.
\end{abstract}

DOI: 10.1103/PhysRevSTAB.7.064202

PACS numbers: 29.27.-a, 41.75.-i, 41.85.Ja

\section{INTRODUCTION}

In high-intensity hadron linacs, parametric 2:1 resonances between coherent oscillations of a mismatched beam core and single particle oscillations are regarded as one of the major sources of beam halo. In tracking studies parametric halo is usually induced using initial mismatch, often in the form of explicit beam eigenmodes. For 1D, 2D, and 3D beams one finds one, two, or three core eigenmodes with distinct eigenfrequencies which have been derived for instance in [1] or [2]. Energy transfer from the core to the single particle oscillations [3,4] can drive core particles to large amplitude radii and thus create a low-density halo surrounding the core. This energy transfer can be studied with a simple particle-core model, where single test particles interact with the space charge forces of the core and the external focusing forces of a continuous focusing channel. In the following this approach is used to introduce the workings of initial mismatch and the development of parametric beam halo. Subsequently, the particle-core model is extended to show that statistical gradient errors, which could also be described as "distributed mismatch," can also trigger the development of beam halo. There are strong indications that the mechanism responsible for expelling single particles to large radii is the same as in the case of initial mismatch. It will be shown that statistical errors result in similar, though much more irregular, core oscillations to the case of initial mismatch [5] and the differences and similarities between the two processes will be outlined in the following. The mechanism of halo development due to statistical errors can be especially important for the understanding of halo in ring systems where the effects of initial or distributed mismatch are generally not taken into account.

\section{PARTICLE-CORE MODEL}

The particle-core model used here follows the approach taken in [6] to derive maximum amplitudes for para-

*Electronic address: f.gerigk@rl.ac.uk metric halo. An azimuthally symmetric continuous beam in a uniform focusing channel is described by the smooth approximation of the envelope equations. One averages over the external forces of a periodic channel and simplifies the space charge calculation by assuming a uniform spatial distribution of the particles within the core. The transverse equation of motion for the core radius $r_{c}$ as a function of the axial coordinate $z$ is thus given by

$$
\frac{d^{2} r_{c}}{d z^{2}}+k_{0}^{2} r_{c}-\frac{\varepsilon^{2}}{r_{c}^{3}}-\frac{K}{r_{c}}=0,
$$

where $k_{0}$ represents the transverse focusing forces as well as the zero-current phase advance per unit length. $K$ is a space charge constant containing mass, axial velocity, charge, and the number of core particles. Together with $k_{0}$ it defines the depressed phase advance per unit length $\left[k^{2}=k_{0}^{2}-\left(2 K / r_{0}^{2}\right)\right] . \varepsilon$ is the total unnormalized emittance. For a matched beam the core radius remains constant $\left(\Rightarrow d^{2} r_{c} / d z^{2}=0\right)$ and one obtains the matched-beam radius as

$$
r_{c}^{2}=r_{0}^{2}=\left(\frac{\varepsilon}{k_{0}}\right)\left[u+\sqrt{1+u^{2}}\right] \quad \text { with } u=\frac{K}{2 \varepsilon k_{0}} .
$$

For a single particle, moving radially in the field of the core, the transverse equation of motion can be written as

$$
\frac{d^{2} x}{d z^{2}}+k_{0}^{2} x-F_{\mathrm{sc}}=0,
$$

where $F_{\mathrm{sc}}$ represents the space charge forces acting on the single particle at radius $x$. Assuming the core as an infinitely long cylinder of uniform charge, particles experience linear forces inside the core and nonlinear forces when leaving the core area:

$$
F_{\mathrm{sc}}= \begin{cases}K x / r_{c}^{2}: & |x|<r_{c}, \\ K / x: & |x| \geq r_{c} .\end{cases}
$$

Outside the core, according to Gauss's law, the space charge forces acting on the particles no longer depend on the actual size of the core. By using particles with different initial radii in the range of $0<x<n r_{c}$, the 


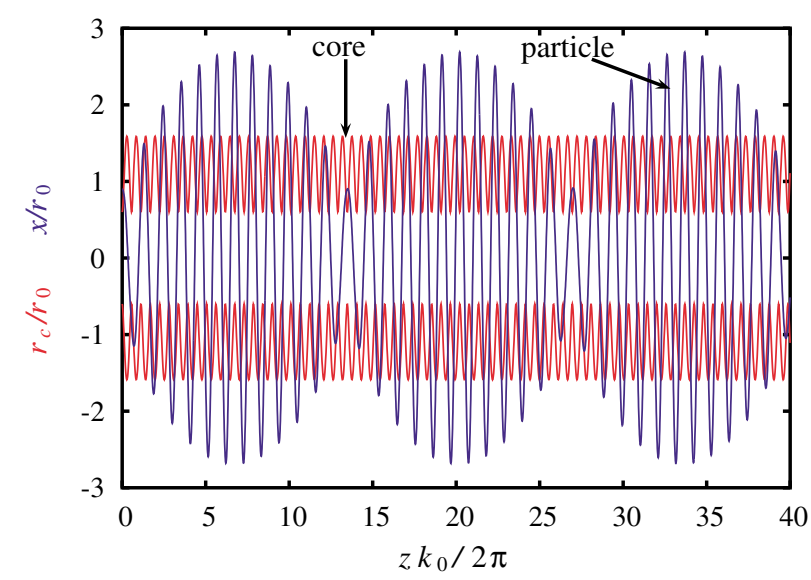

FIG. 1. (Color) Particle and core envelopes for initial mismatch, $\mu=0.6, x(0)=0.9 r_{0}$, and $\tau=0.7$.

particle-core model is used to study the circumstances under which particles can be driven to large amplitude oscillations. In all of the following simulations the initial transverse momenta of the single particles are set to zero.

\section{A. Initial mismatch}

In the matched case the core radius as well as the amplitudes of the single particle oscillations remains constant. If the core is mismatched initially $(\mu=$ $r_{c} / r_{o} \neq 1$ ), its radius starts to oscillate around its equilibrium value $r_{0}$. In our simple 1D model an initial radial mismatch corresponds to the excitation of the breathing mode (fast mode), which is the only beam eigen mode for an azimuthally symmetric, continuous beam. Single particles traversing the core can obtain a net energy gain if the core radius is different for a particle during entering and leaving the core. As an example we integrate the equations of motion for a tune depression of $\tau=k / k_{0}=$ 0.7 , a core mismatch of $\mu=0.6$, and an initial single particle radius of $0.9 r_{0}$ (see Fig. 1).

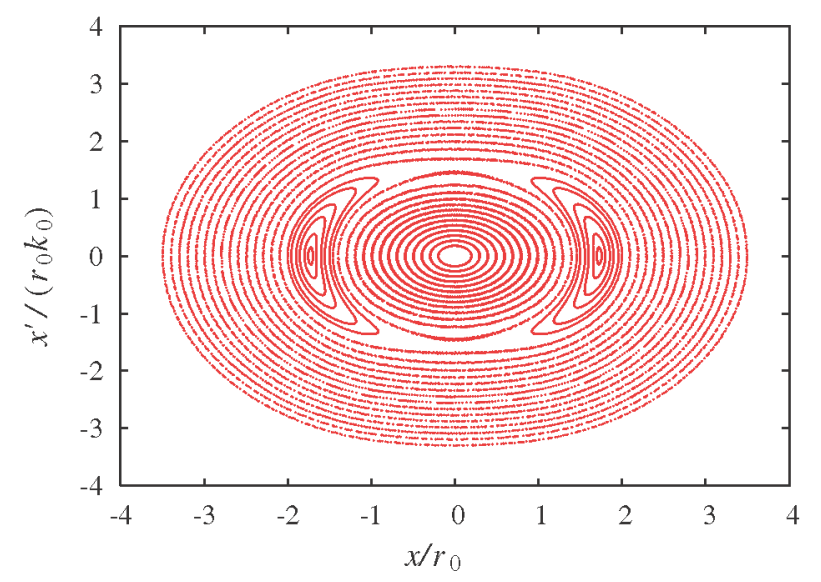

FIG. 2. (Color) Stroboscopic plot for $\mu=0.95, \quad 0.2 \leq$ $x(0) / r_{0} \leq 3.5$, and $\tau=0.8$.
If the oscillation frequency of the single particle has a 1:2 parametric ratio with the oscillation frequency of the core, the particle gains energy and increases its oscillation amplitude until the resonant condition is no longer fulfilled. As a result one finds a certain maximum for the halo radius as well as so-called fixed points in phase space around which halo particles start to conglomerate. The maximum halo radius is determined by the amount of initial mismatch, while the time constant for the development of beam halo is influenced by the tune depression [6]. The position of the fixed points seems to be fairly insensitive to changes in tune depression or mismatch amplitude. In [7], however, it was shown that the fixedpoint core distance changes for nonequipartitioned beams $\left(\varepsilon_{t} k_{t} \neq \varepsilon_{l} k_{l}\right)$. To visualize the fixed points as well as the maximum halo amplitudes one uses stroboscopic plots showing the phase space position of the single particle once per core-oscillation period. For Figs. 2 and 3 the transverse momentum and position of the particle is plotted every time the core oscillation reaches its minimum, which coincides with the occurrence of the maximum single particle radii (compare Fig. 1). To obtain a coherent resonance pattern a large number of focusing periods is needed (4000 in this example). Each dotted line represents a single particle with a certain initial radius and zero initial transverse momentum. In the case of weak initial mismatch (e.g., 5\%, Fig. 2) particles that start within the core $\left(x / r_{0} \leq 1\right)$ remain basically undisturbed in their trajectory. They either need a nonzero initial momentum or an initial radius larger than the core radius to gain energy by means of a parametric resonance. In contrast, for strong initial mismatch (e.g., $40 \%$, Fig. 3), the resonant regions surrounding the fixed points become very large and the core area where particles remain undisturbed in their trajectories shrinks considerably. In both cases particles with large initial radii, exceeding the resonant areas around both fixed points, remain as unperturbed in their orbits as the inner

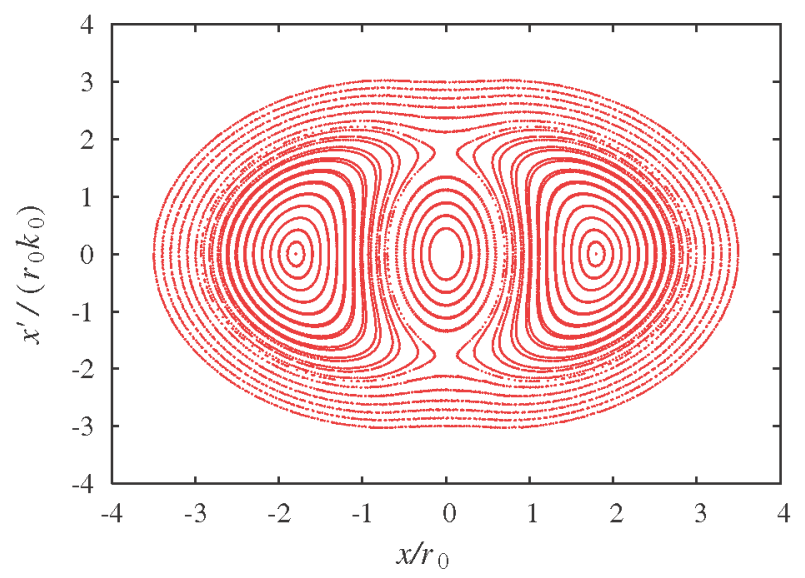

FIG. 3. (Color) Stroboscopic plot for $\mu=0.6,0.2 \leq x(0) / r_{0} \leq$ 3.5 , and $\tau=0.8$. 
core particles. This pattern can be explained by remembering that the net energy gain for the single particles is determined by the different core sizes, when particles enter and leave the core region. As a consequence, particles with very large amplitudes, which spend only a small fraction of their trajectory inside the core, do not obtain significant energy kicks, since they cannot enter into a resonance with the core oscillation. The same is true for particles with small initial radii which remain within the (oscillating) core volume, even if their trajectories change slightly. Most affected are the particles "in between" which cross the core boundaries very often and which are then "caught" in a parametric resonance.

Assuming that all particles are initially confined within the core, this classic 1D particle-core model predicts maximum halo radii of $\approx 3 r_{0}$. It is worth noting that particles which are initially outside the core and get into resonance with the core oscillations have the same maximum halo radius as particles starting inside the core. Studies with a more sophisticated 3D particle-core model [8] show that the maximum halo extent due to initial mismatch can amount to 5 or even more times the radius of the matched beam, if the longitudinal to transverse focusing ratio is larger than 1 .

\section{B. Statistical errors}

To study statistical errors, the focusing constant $k_{0}$ in Eqs. (1) and (3) is modified in every focusing period, simulating the effect of statistically distributed gradient errors in a transport channel. The errors are applied using a Gaussian distribution with a cutoff at twice the rms value. Figure 4 shows the effect of a $1 \%$ (rms) focusing error on the core and the single particle envelopes using an initial single particle radius of $x(0) / r_{c}=1.2$. After a certain number of periods the core starts oscillating in a similar manner as for initial mismatch. Because of the irregular excitation of the core its oscillation amplitude is

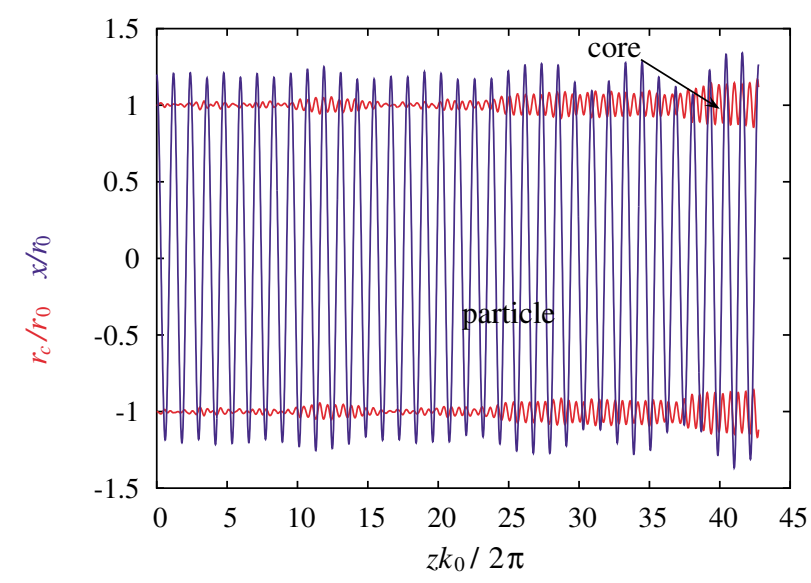

FIG. 4. (Color) Particle and core envelopes for a matched beam with $1 \% \mathrm{rms}$ statistical focusing gradient errors $x(0) / r_{0}=1.2, \tau=0.8$. now subject to a slowly changing random modulation. The oscillation frequency, however, remains almost constant at the frequency of the eigenmode and only changes within a few per cent around this reference value. Only for very small oscillations, as in the first periods of Fig. 4, can the oscillation frequency change by up to $\approx 20 \%$.

Even though the errors are statistically distributed, the core oscillations can reach considerable amplitudes, a phenomenon that has also been observed in realistic linac simulations [5]. Since the core oscillations are irregular as opposed to those resulting from initial mismatch (e.g., Fig. 1), it is unlikely for single particles to enter a stable parametric resonance for more than a few oscillation periods. Nevertheless, there are regions where the core oscillations maintain an almost constant amplitude (e.g., periods 25-40 in Fig. 4), and during these it seems that the single particle enters a similar oscillation pattern as for initial mismatch in Fig. 1: the maximum amplitude of the single particle is rising and falling with a more or less sinusoidal modulation. This pattern suggests that even a few oscillation periods which are more or less "in phase" suffice to transfer energy from the core to the single particle trajectories. Comparing once more with the case of initial mismatch in Fig. 1 this explanation seems very likely, since also there the energy transfer practically starts from the first few core oscillations onwards, implying that the mechanism to transfer energy is the same as for initial mismatch. In the case of initial mismatch the core immediately oscillates with a large amplitude yielding an increase of single particle radii by a factor of $2-3$ within a few periods. For statistical errors, however, the particle-core model suggests that several hundred periods can be necessary to achieve core-oscillation amplitudes that are large and stable enough to result in comparable single particle radii.

Plotting the maximum and minimum values of core and single particle envelopes along the channel for different initial single particle radii (Fig. 5) confirms that the single particle envelopes are oscillating in a manner similar to initial mismatch. It also shows that particles from within the core boundaries are only weakly affected, whereas particles starting outside of the core boundaries show a clear increase in their oscillation amplitudes.

\section{Average effects}

In order to show which particles experience on average the largest amplitude growth, Figs. 6-8 show the growth factors for single particle amplitudes as a function of their initial radius. Each of the plotted points represents the average of maximum amplitude values found in 1000 simulations with different error sets. The curves connecting the points are interpolated with cubic splines.

Figure 6 shows that for an emittance dominated beam $(0.71<\tau<1.0)$ the maximum growth factors (or halo 


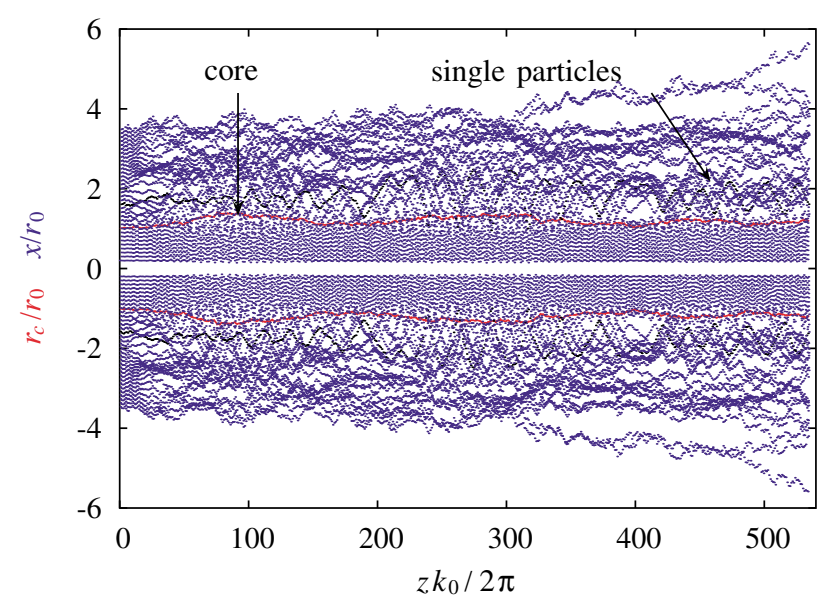

FIG. 5. (Color) Maximum envelope values for core and single particle oscillations in the case of a matched beam with $1 \% \mathrm{rms}$ statistical focusing gradient errors $0.2 \leq$ $x(0) / r_{0} \leq 3.5, \tau=0.8$.

amplitudes) clearly depend on the tune depression of the beam, while they remain basically constant for space charge dominated beams $(0.0<\tau<0.71)$. The same observation was made in [6] for halo development due to initial mismatch.

Figure 7 shows, not surprisingly, that the maximum oscillation amplitudes depend on the amplitude of the statistical variation that is applied to the focusing forces, and finally, Fig. 8 explores how the maximum amplitude growth depends on the length of the simulated transport channel.

In all three cases (Figs. 6-8) the maximum amplitude growth is found for particles with initial radii around $1.5 r_{0}$, suggesting that these particles have the highest probability of entering a parametric resonance with the

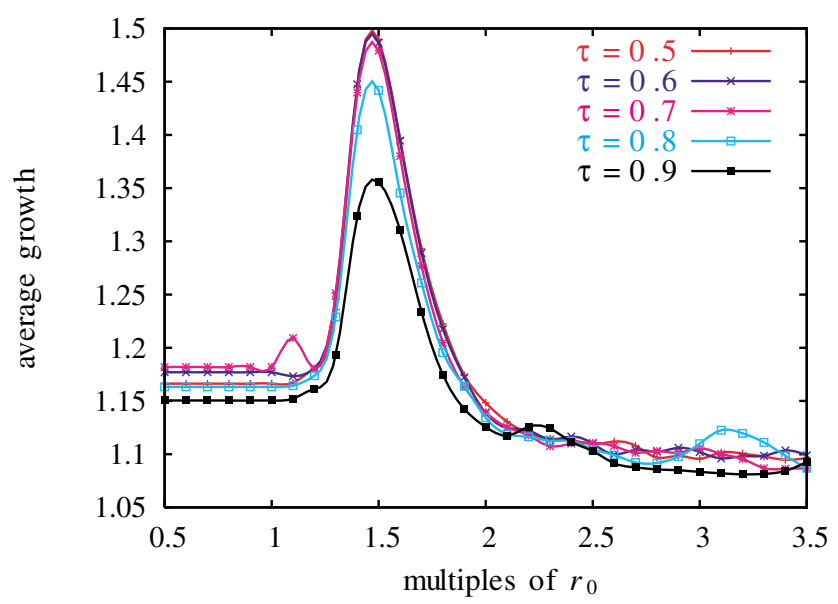

FIG. 6. (Color) Maximum amplitude growth for single particles over 100 zero-current betatron periods as a function of their initial radius and tune depression $\tau$. Each dot represents the averaged results of 1000 runs with different error sets for $1 \%$ rms error.

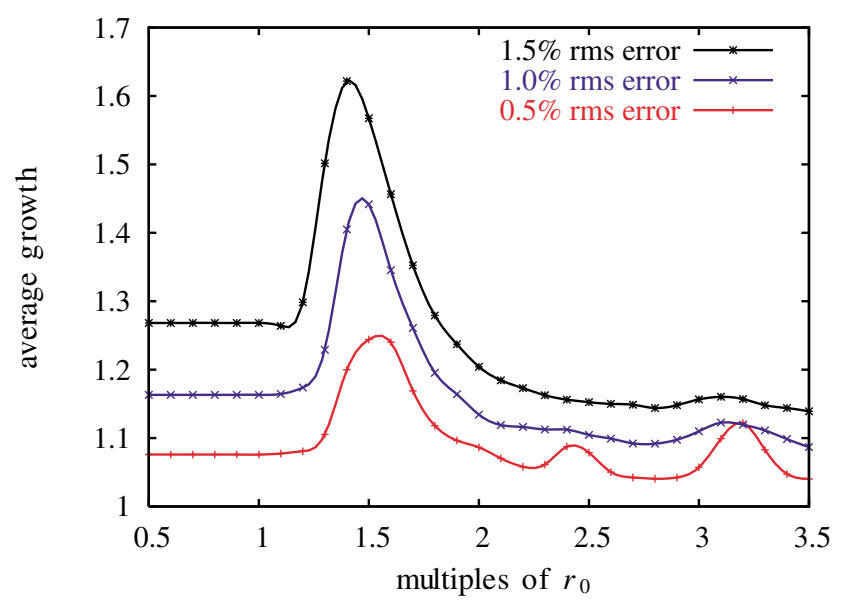

FIG. 7. (Color) Maximum amplitude growth for single particles over 100 zero-current betatron periods as a function of their initial radius and of the rms error amplitude. Each dot represents the averaged results of 1000 runs with different error sets for a tune depression of $\tau=0.8$.

core oscillation. In order to show that the underlying mechanism is indeed based on a resonance, Fig. 9 shows the wave numbers (or tunes per meter) for the core $\left(k_{\text {core }}\right)$ and the single particle ( $\left.2 k_{\text {particle }}\right)$ oscillations, assuming one random error set with different initial radii for the single particle. The data are approximated by bezier curves to reduce the amplitudes of the rapidly changing wave numbers and to obtain smooth and easily distinguishable curves.

After 20-30 zero-current betatron periods the core wave number assumes a relatively stable value of $\approx 3.7$. A few periods later the wave numbers of particles, that start with an initial radius between 1.3 and 2.1 times the

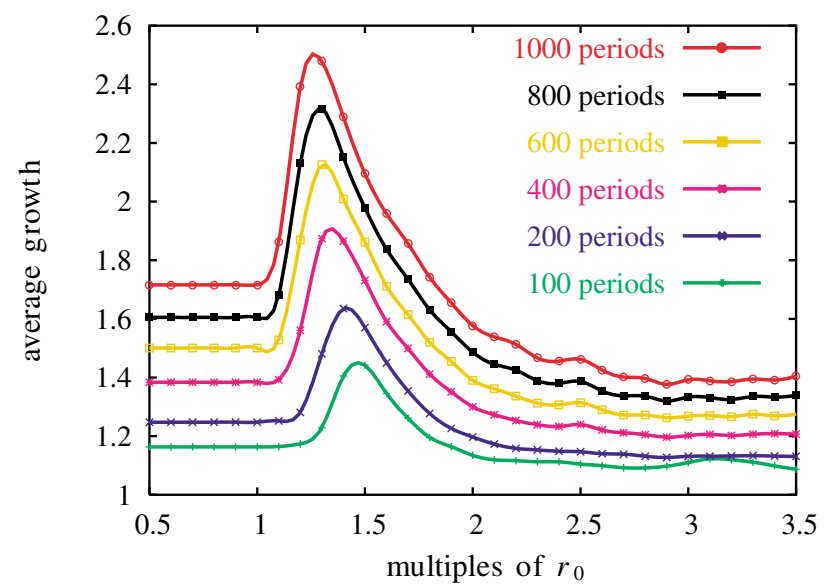

FIG. 8. (Color) Maximum amplitude growth for single particles as a function of their initial radius and the length of the transport channel (in zero-current betatron periods). Each dot represents the averaged results of 1000 runs with different error sets for a tune depression of $\tau=0.8$ and an rms error amplitude of $1 \%$. 


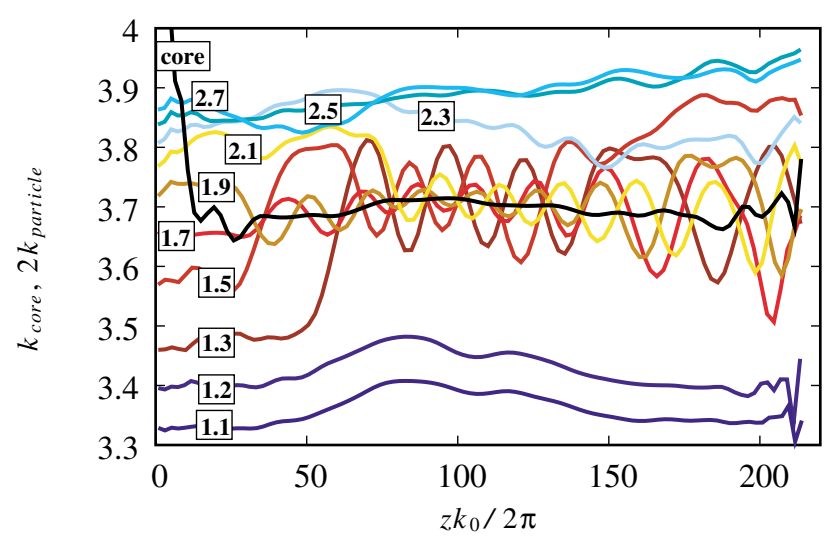

FIG. 9. (Color) Wave numbers for core and single particle oscillations. Single particle wave numbers are multiplied by 2 and depict particles starting with different initial radii (1.1-2.7 times the matched core radius), $\tau=0.8$, rms error amplitude: $1 \%$.

matched core radius, begin to oscillate around an average that is half as big as the core wave number, meaning that they fulfill the condition for a parametric 1:2 resonance with the core. It is interesting to note that particles whose initial wave numbers are very close to 0.5 times the core wave number start very quickly to resonate with the core, while it takes more time until particles with larger or smaller wave numbers are affected. Particles that start with initial radii $\leq 1.2 r_{0}$ or $\geq 2.3 r_{0}$ have either too small or too high a wave number to enter a resonance within the length of the calculated transport channel.

We expect that the range of particles likely to interact with the core oscillations will be much larger in a more realistic 3D beam with altogether three different core eigenfrequencies. Furthermore the maximum amplitude values and their associated time constants will certainly be different for more realistic beams with nonuniform distributions and nonlinear space charge forces within the core area.

Even though one could imagine that for statistical errors, the particles experience on average as much negative energy kicks as positive kicks, Fig. 8 clearly shows that statistical errors have a cumulative effect. This can be understood by interpreting statistical errors as a continuous supply of "free energy" which can only increase the transverse beam temperature (or energy) but never yield a decrease (compare $[9,10])$. In the case of initial mismatch the beam obtains one large energy kick at the beginning and the particles as well as the core perform regular oscillations around their equilibrium. Without further disturbance these oscillations are maintained indefinitely in the particle-core model, while a continuous supply of "energy" via statistical errors yields ever increasing particle amplitudes. We note at this point that the core oscillations of a realistic 3D beam, caused by initial mismatch, are eventually damped by the energy transfer from the core to the single particle oscillations. This transformation from mismatch to beam halo and rms emittance growth has been found to develop much more rapidly for Gaussian beams than for the idealized 6D waterbag beams (compare Fig. 14 and also [11]).

\section{Halo development}

While the average effects give a good indication of the general particle behavior due to statistical errors, they provide little information about the development of beam halo and its maximum extent in phase space. To illustrate the formation of halo driven by statistical errors we use the same kind of stroboscopic plot that showed the resonance pattern for initial mismatch (e.g., Fig. 3). Figure 10 depicts the pattern of one particle for one particular error set, which has a larger than average amplitude growth. One can see that initially the particle maintains a phase space trajectory close to the core boundaries. At some point the particle receives a kick that, within a few periods, rapidly increases its maximum radius from $\approx 2 r_{0}$ to $\approx 3.5 r_{0}$, where it remains for another $\approx 150$ zero-current betatron periods. Later on another kick expels the particle even further to radii $>4 r_{0}$. Contrary to initial mismatch, particles seem to increase their maximum radii further and further, provided the length of transport channel is long enough.

To assess more generally the potential for halo development we evaluate in Fig. 11 the probability for particles to reach large amplitudes as a function of their initial radius and the length of the transport channel.

Particles that start at $1.2<x(0) / r_{0}<2.1$ show an almost equal probability of reaching certain large amplitudes, which can be explained by the enhanced amplitude growth that was observed earlier for particles with initial radii around $\approx 1.5 r_{0}$. The plots also confirm the extremely low probability for particles starting from within the core to actually transgress the core boundaries.

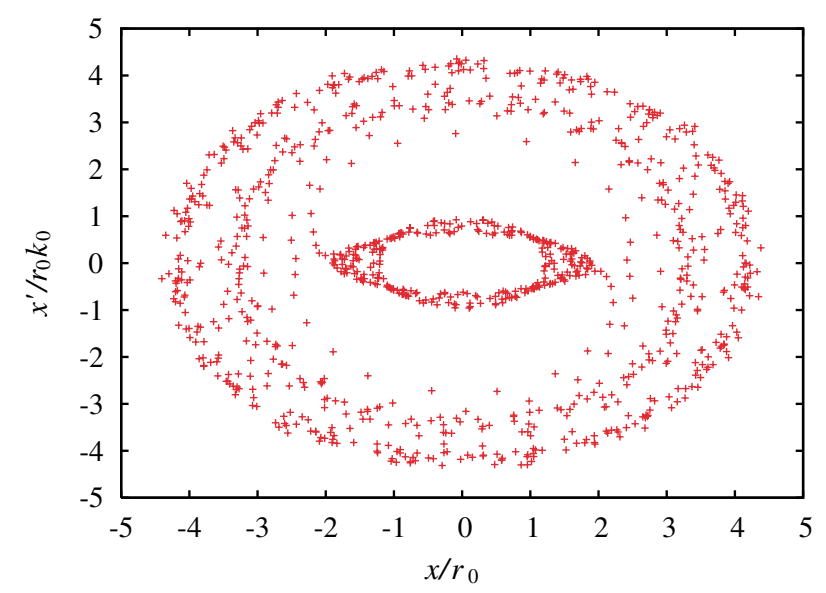

FIG. 10. (Color) Stroboscopic plot for a single particle with $x(0) / r_{0}=1.2,1 \% \mathrm{rms}$ error, and 1000 zero-current betatron periods. 

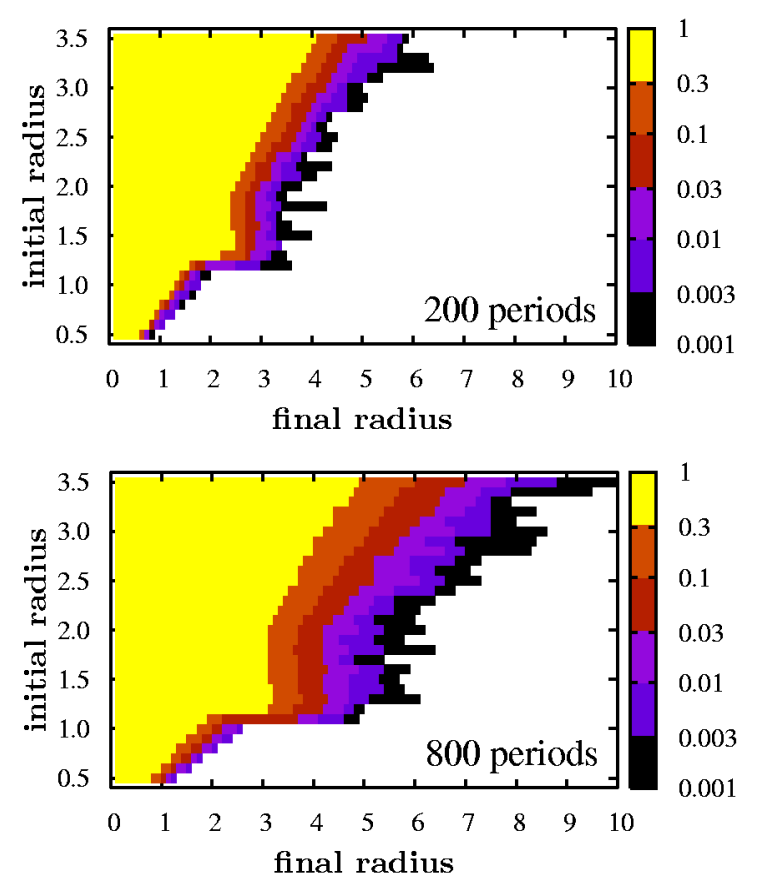

FIG. 11. (Color) Probability for single particles to reach final radii with large amplitudes; 200 and 800 zero-current betatron periods. The black bars represent one out of a total of 1000 simulations.

\section{Limitations of and conclusions from the particle- core model}

A continuous, azimuthally symmetric, unbunched beam in a constant focusing channel is a very crude approximation for a realistic 3D linac beam propagating through a periodic, accelerating lattice which is subject to the influence of nonlinear forces. However, as a proof of principle, the model shows that halo development can be triggered by statistical errors and that the process of expelling single particles to large amplitudes is based on a parametric resonance. It also suggests that in a space charge dominated beam the maximum halo amplitudes are independent of the tune depression. Furthermore it shows that the maximum radii depend on the error amplitudes and on the length of the transport channel. Contrary to initial mismatch there seems to be no boundary for the extent of the halo produced, suggesting that particles will inevitably get lost even if one uses accelerating lattices that allow large bore radii (e.g., superconducting cavities). The model cannot predict rms emittance growth and allows only very limited conclusions about the time constants for halo development along with the associated maximum amplitudes. While the time constants for single particles to become halo particles might seem very long, the situation certainly changes when considering the large number of particles in a realistic beam. There, even a tiny fraction of particles $\left(10^{-5}-10^{-7}\right)$ acquiring large radii may cause losses that limit the maximum machine currents.
TABLE I. Parameters for the transport channel in the particle-core model.

\begin{tabular}{lcc}
\hline \hline Tune depression $(\tau)$ & $0.5-0.9$ & \\
Energy & 11.4 & $\mathrm{MeV}$ \\
Focusing period $(l)$ & 0.333 & $\mathrm{~m}$ \\
Zero-current phase advance & & \\
$\quad$ per period $\left(l k_{0}\right)$ & 38.5 & $\mathrm{deg}$ \\
\hline \hline
\end{tabular}

\section{3D PARTICLE TRACKING}

To study the effects of statistical errors on a realistic 3D beam we use a periodic focusing channel without acceleration and with the same basic properties as used for the particle-core model (see Table I). For each case considered in the following, 500 randomly created error sets (Gaussian error distribution with cutoff at twice the rms value) are simulated with IMPACT [12], using $10^{5}$ particles.

Figure 12 characterizes the output distribution for transport channels of different lengths, showing the average fraction of particles exceeding a certain multiple of the initial transverse rms emittance $\left[\varepsilon_{t}=\left(\varepsilon_{x}+\varepsilon_{y}\right) / 2\right]$.

The fraction of particles exceeding the initial distribution increases approximately linearly with the length of the transport channel. In terms of the "free energy approach" one can argue that statistical errors represent a continuous feed of "free energy" into the system, which is transformed into rms emittance growth and beam halo.

As predicted by the particle-core simulations, the maximum halo amplitudes in the case of statistical errors can reach significantly higher radii than in the case of initial mismatch. The probability, however, of reaching radii in excess of 10 times the rms radius or more, seems very low. Nevertheless, the simulations suggest that the

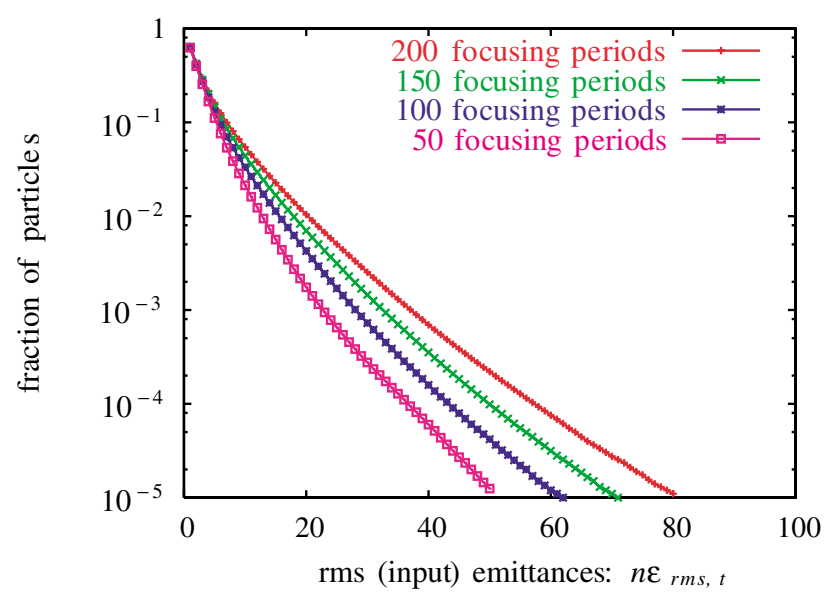

FIG. 12. (Color) Averaged fraction of particles exceeding $n \varepsilon_{\mathrm{rms}, t}$ for transport channels of different length (100 focusing periods correspond to $\approx 10.7$ zero-current betatron periods), 6D Gauss with 1\% (rms) quadrupole gradient errors, 500 simulations, normalized to transverse input emittance. 


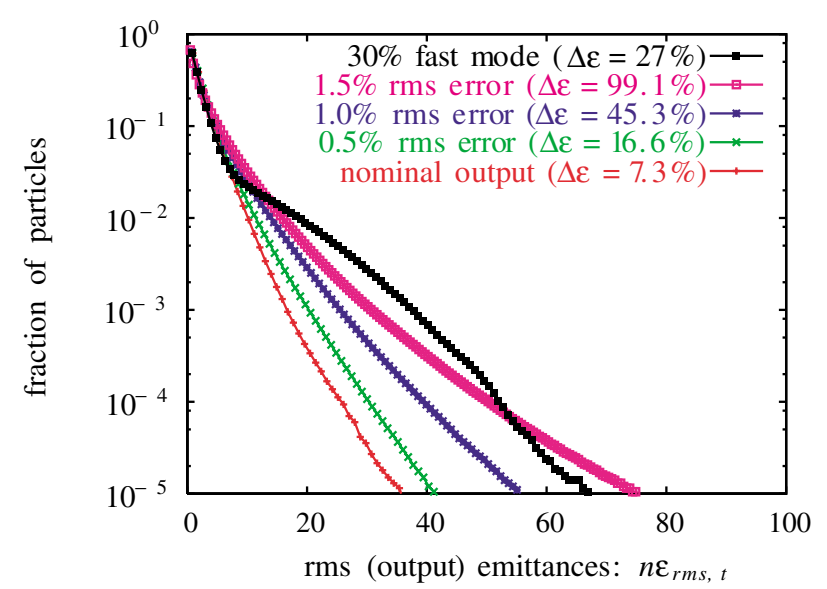

FIG. 13. (Color) Averaged fraction of particles exceeding $n \varepsilon_{\mathrm{rms}, t}$ for statistical error runs normalized to the final rms emittance of each case. 500 simulations, 6D Gauss, 200 focusing periods, and $\Delta \varepsilon \rightarrow$ rms emittance growth.

effects of statistical errors could be seen in linear accelerators, which have a high number of focusing elements in their low-energy sections. Below $150 \mathrm{MeV}$ one finds approximately between five and ten zero-current betatron periods in a typical normal conducting linac $(\approx 200$ quadrupoles plus $300 \mathrm{rf}$ gaps), which, depending on the lattice characteristics, may already be long enough to yield losses due to statistical errors. Using for instance only five zero-current betatron periods with a $1 \% \mathrm{rms}$ gradient error (Fig. 12), one already finds $10^{-5}$ of the particles beyond $50 \mathrm{rms}$ input emittances which corresponds to $\approx 7 \mathrm{rms}$ radii.

In synchrotrons or storage rings for space charge dominated beams, where the bunches are transported through a large number of lattice periods, statistical errors may well account for the development of substantial parametric beam halo that has to be scraped by dedicated beam collimation systems. In order to show that statistical errors not only increase the rms emittance but do in fact produce a low-density beam halo, we plot in Fig. 13 the averaged fraction of particles exceeding certain multiples of the transverse rms emittance for three different rms error amplitudes and normalize each curve by its output rms emittance, thus removing the rms emittance growth from the plots.

Compared with a 30\% (amplitude) excitation of the fast mode we find that, on average, the effects of statistical errors are much less dramatic. For the fast mode excitation one can observe a certain "hump" in the output distribution which is probably a result of the very stable oscillation of the mismatched beam core. For statistical errors the resonant conditions are changing very rapidly and thus the output distributions become very smooth. In the case of initial mismatch one usually finds that Gaussian distributions develop beam halo more quickly than waterbag distributions.
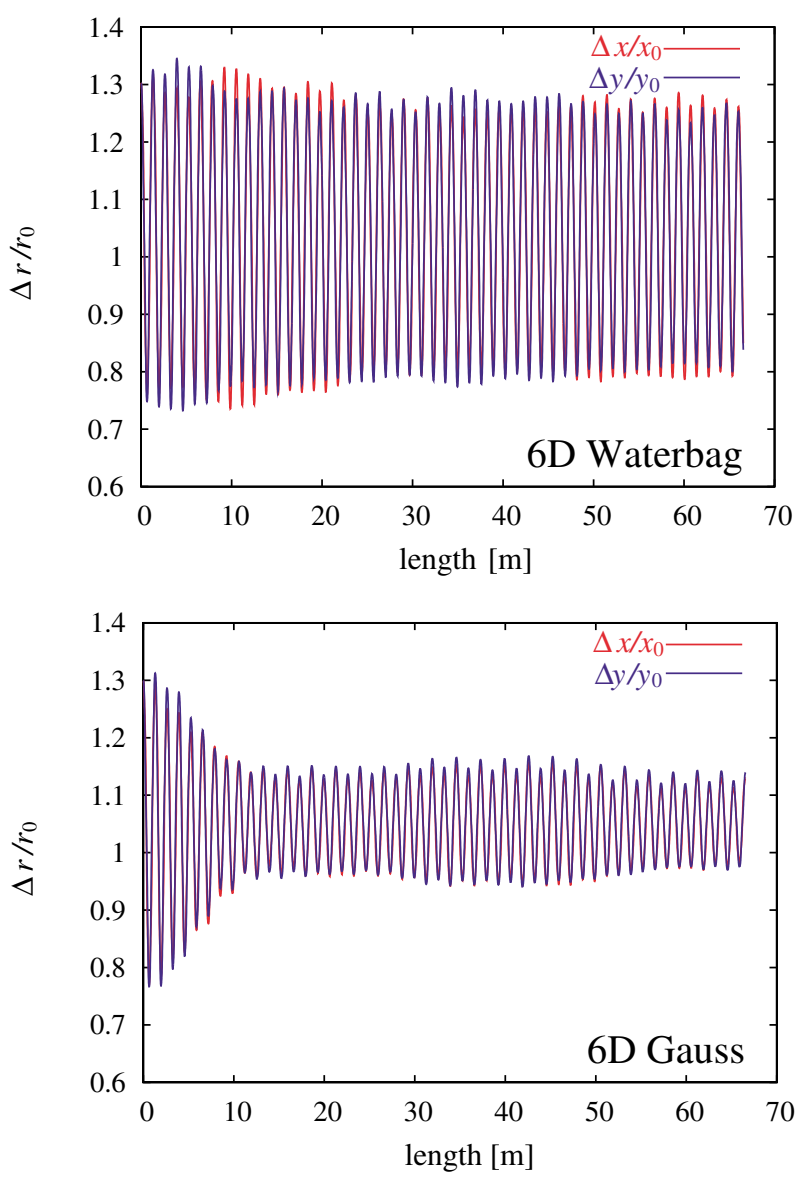

FIG. 14. (Color) Mismatched transverse core radii over matched core radii for 6D waterbag and Gauss, 200 focusing periods, and fast mode excitation.

In Fig. 14 one can see that the transverse rms core oscillations for a Gaussian beam with $30 \%$ fast mode excitation are rapidly damped from $30 \%$ to $\approx$ $12 \%$ where they remain almost constant. At the same

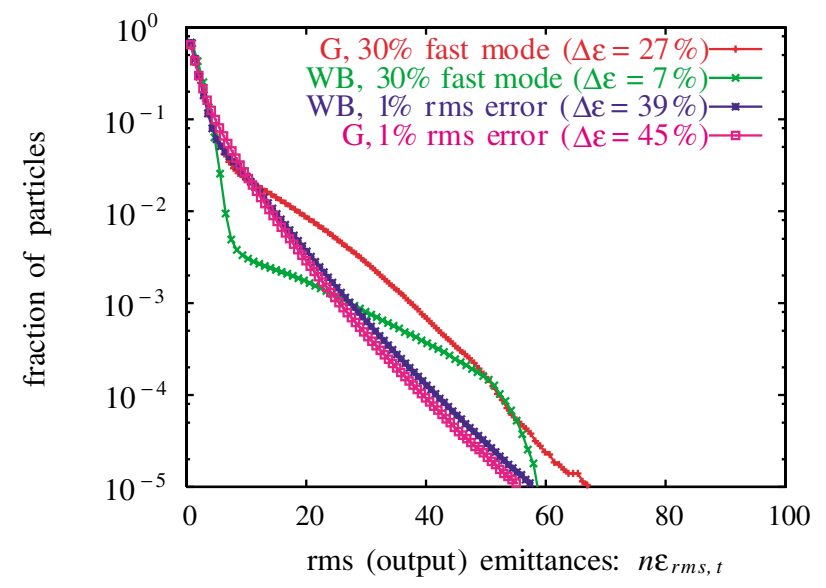

FIG. 15. (Color) Averaged fraction of particles exceeding $n \varepsilon_{\mathrm{rms}, t}$ for statistical error runs normalized to the final rms emittance of each case. 500 simulations, 6D Gauss/waterbag, 200 focusing periods, and $\Delta \varepsilon \rightarrow$ rms emittance growth. 
time the waterbag core loses only a fraction of its oscillation energy with the result of much smaller emittance growth (7\% versus $27 \%$ for 6D Gauss) and halo development in a transport channel of equal length. For statistical errors we find that there not such a distinct difference between the two distributions. Figure 15 shows the averaged fraction of particles exceeding a certain multiple of the rms emittance for simulations with initial waterbag and Gaussian distributions using initial mismatch and/or statistical errors.

While in the case of initial mismatch the difference between the Gaussian and the waterbag beam $(27 \% \mathrm{rms}$ emittance growth versus $7 \%$ ) is clearly visible; only a very small difference can be observed for statistical errors. This is indeed quite surprising since the particle-core model seems to suggest that those particles mainly affected by statistical errors are initially outside the core.

\section{CONCLUSIONS}

Using a simple particle-core model one can show that statistical errors can excite oscillations of the beam core which can then, via parametric resonances, transfer energy to single particles. 3D simulations for a periodic transport channel show that this mechanism not only results in rms emittance growth but also initiates the development of a low-density beam halo surrounding the core. The process scales more or less linearly with the error amplitude and the length of the transport channel but seems to be almost insensitive to the type of particle distribution that is chosen in $3 \mathrm{D}$ simulations. For emittance dominated beams the effect depends on the level of tune depression and reaches its maximum when entering the space charge dominated regime $(\tau<0.7)$ where no further dependency on the tune depression is observed.

For most simulations a $1 \% \mathrm{rms}$ gradient error was applied, which may seem high in view of the specifications for new linac projects like the Spallation Neutron Source $(0.14 \%$ rms quadrupole gradient error [13]). Nevertheless, most of todays operating accelerators do not fulfill such tight margins and one should keep in mind that quadrupole gradient errors are only one error type that has to be added to a whole range of error sources. However, for high-intensity cw linacs or machines with very high duty cycles (e.g., linacs as fusion drivers or for waste transmutation) even much smaller error margins may not be sufficient to prevent the development of unwanted and harmful beam halo. As a rule of thumb we find that the effect of a $1 \%$ rms quadrupole gradient error in terms of emittance growth and halo development is comparable to the effect of a $20 \%$ initial fast mode excitation (assuming a transport channel of $\approx 15$ zerocurrent betatron periods and a space charge dominated beam). In circular accelerators, statistical errors provide a continuous source for halo development as long as the beam is in a space charge dominated regime. Here, they may at least partly explain the characteristic "halo shoulders" that can be observed in most synchrotrons and that have to be controlled by beam collimation systems.

\section{ACKNOWLEDGMENTS}

We acknowledge the support of the European Community-Research Infrastructure Activity under the FP6 "Structuring the European Research Area" program (CARE, Contract No. RII3-CT-2003-506395).

[1] K. Bongardt, A. Letchford, and M. Pabst, in Proceedings of the 1999 Particle Accelerator Conference, New York (IEEE, Piscataway, NJ, 1999).

[2] N. Pichoff, CEA Report No. DAPNIA/SEA 98/44, 1998.

[3] J. O'Connell, T. Wangler, R. Mills, and K. Crandall, in Proceedings of the 1993 Particle Accelerator Conference, Washington, DC (IEEE Report No. CH 3279-7, 1993), pp. 3657-3659.

[4] R. Gluckstern, Phys. Rev. Lett. 73, 1247 (1994).

[5] F. Gerigk, in Proceedings of the 2003 Halo Workshop, Montauk, AIP Conf. Proc. No. 693 (AIP, New York, 2003), pp. 61-64.

[6] T. Wangler, K. Crandall, R. Ryne, and T. Wang, Phys. Rev. ST Accel. Beams 1, 084201 (1998).

[7] G. Franchetti, I. Hofmann, and D. Jeon, Phys. Rev. Lett. 88, 254802 (2002).

[8] J. Qiang and R. Ryne, Phys. Rev. ST Accel. Beams 3, 064201 (2000).

[9] M. Reiser, J. Appl. Phys. 70, 1919 (1991).

[10] G. Franchetti and I. Hofmann, in Proceedings of the 2002 European Particle Accelerator Conference, Paris, France (EPS-IGA/CERN, Geneva, 2002).

[11] I. Hofmann, G. Franchetti, J. Qiang, R. Ryne, F. Gerigk, D. Jeon, and N. Pichoff, in Proceedings of the 2002 European Particle Accelerator Conference, Paris, France (Ref. [10]).

[12] J. Qiang, R. Ryne, S. Habib, and V. Decyk, J. Comput. Phys. 163, 434 (2000).

[13] J. Galambos, I. Anderson, N. Holtkamp, and C. Strawbridge, SNS Parameter List Report No. SNS 100000000-PL0001-R09, 2003. 